\title{
Utilização de estratificação e modelo de regressão logística na análise de dados de estudos caso-controle
}

\author{
Using of stratitification and the logistic regression model \\ in the analysis of data of case-control studies
}

\author{
Suely Godoy Agostinho Gimeno, José Maria Pacheco de Souza \\ Departamento de Epidemiologia da Faculdade de Saúde Pública. \\ Universidade de São Paulo - São Paulo, SP - Brasil
}

\begin{abstract}
Exemplifica-se a aplicação de anállse multivariada, por estratíficaçăo e com regressăo logística, utilizando dados de um estudo caso-controle sobre cáncer de esófago. Oitenta e cinco casos a 292 controles foram classificados segundo sexo, ldade e os hábitos de beber e de fumar. As estimatlvas por ponto dos od ds ratios foram semelhantes, sendo as duas técnicas consideradas complementares.
\end{abstract}

Análise multivariada. Regressäo logistica. Estudos de casos e controles.

EmEpidemiologia, a regressão logística tem como objetivo descrever a relaçâo entre um resultado (variável dependente ou resposta) e um conjunto simultâneo de variáveis explicativas (preditoras ou independentes), mediante um modelo que tenha bom ajuste, que seja biologicamente plausível e obedeça ao princípio da parcimônia ${ }^{5}$. Na análise estratificada tem-se o mesmo propósito, mas as relaçōes são efetuadas uma a uma, isto é, somente é possível obter a estimativa do risco para um único fator de cada vez, controlando-se o conjunto das demais variáveis.

Podem ser citadas como vantagens da análise estratificada sua relativa simplicidade de execução, a facilidade de entendimento e maior proximidade que propicia entre o pesquisador e os dados. Entretanto, ao se aplicar essa técnica, o grau de dificuldade aumenta na medida em que aumenta o número de variáveis que tiverem que ser consideradas como controle; os testes de homogeneidade entre os estratos, para se verificar a existência de interação entre as variáveis, são feitos em etapa à parte; variáveis quantitativas não podem ser usadas em sua escala original; o nível global de significância é difícil de ser controlado ${ }^{3,7}$.

A análise logística controla grande número de variáveis simultaneamente, permitindo que os dados sejam utilizados mais eficientemente; o teste de homogeneidade pode ser feito em conjunto, bastando introduzir no modelo o termo produto entre os fatores. Uma possível desvantagem é a eventual barreira que introduz entre o pesquisador e os dados; é praticamente obrigatório o uso de pacotes estatísticos e microcomputador ${ }^{4,5}$.

$O$ presente trababalho exemplifica a utilização das análises estratificada e logística na análise de dados de estudos tipo caso-controle.

\section{Material e Método}

Foram utilizados dados de um estudo caso-controle sobre câncer de esôfago ${ }^{6}$. Oitenta e cinco casos de câncer de esôfago foram comparados com 292 controles hospitalares, classificados segundo segundo sexo, idade e os hábitos de beber e de fumar. O hábito de beber foi considerado fator de risco de principal interesse.

Foi verificada a existência de associação entre o câncer de esôfago e cada uma das variáveis, em uma primeira etapa (análise bruta), mediante a obtenção das estimativas dos odds ratios (OR), por ponto $\mathrm{e}$ por intervalo, além do valor da estatística quiquadrado de Mantel-Haenszel $\left(\chi_{\text {s+1 }}^{2}\right)^{1,2,9,10}$. Nas etapas seguintes foram selecionadas as variáveis cujo 
valor do nível descritivo de significância do teste fosse menor do que $0,20^{5}$.

A análise estratificada foi utilizada na obtençāo da estimativa do odds ratio para o hábito de beber, controlando-se o efeito dos demais fatores previamente selecionados. Fez-se, quando possível, o teste de homogeneidade dos estratos, a fim de investigar a existência de interação entre as variáveis ${ }^{2,9,10}$. Este procedimento foi repetido utilizando-se o modelo de regressāo logística (năo condicional em virtude de não haver emparelhamento); a presença de interação entre as variáveis foi verificada mediante a introdução dos termos-produtos correspondentes. Os resultados das duas técnicas foram comparados.

Nos apêndices encontram-se as formulas utilizadas para a obtençāo das estimativas dos odds ratios, assim como a descriçāo dos testes estatísticos. Foram utilizados os pacotes estatísticos para microcomputador Epi Info ${ }^{3}$ e MULTLR ${ }^{8}$. Os intervalos de confiança para os estratos foram feitos segundo a técnica de Woolf ${ }^{2}$.

\section{Resultados}

A Tabela 1 apresenta a distribuição completa dos casos e controles segundo sexo, idade e os hábitos de beber e de fumar. Na Tabela 2 encontram-se os resultados da análise bruta; a variável idade apresentou valor $p>0,20 \mathrm{e}$, dessa forma, não foi considerada nas etapas seguintes da análise. Nas Tabelas 3, 4, 5, 6 e 7 são apresentados os resultados das análises estratificada e logística, com uma e
Tabela 2- Resultados da análise bruta.

\begin{tabular}{lccc}
\hline Variável & Odds ratio & $\chi_{n+1}^{2}$ & $p$ \\
\hline Hábito de beber & 6,91 & 35,88 & 0,00 \\
Hábito de fumar & 8,93 & 33,09 & 0,00 \\
Sexo & 3,41 & 17,83 & 0,00 \\
Idade & 0,80 & 0,82 & 0,37 \\
\hline
\end{tabular}

duas variáveis como controle.

Na Tabela 3 há sugestão de interação entre os hábitos de beber e de fumar e, também, parece haver açāo protetora da bebida sobre a doença; como, sabidamente, o hábito de beber é importante fator de risco para o câncer de esôfago, torna-se indispensável a visualização dos dados no sentido de explicar o paradoxo. $O$ pequeno número de casos entre os não-fumantes é responsável pela distorçăo observada; bastaria que a relação bebe: não bebe fosse $2: 4$ $\mathrm{e}$ o odds ratio seria 1,34. Na Tabela 4 os resultados são os esperados. $\mathrm{Na}$ análise estratificada, ao se considerar duas variáveis para a estratificaçāo (Tabela 6), não foi possivel fazer o teste de homogeneidade, devido a frequiência zero.

Nas Figuras 1, 2 e 3 estão apresentadas as estimativas, por ponto e por intervalo, dos odds ratios obtidos com a análise estratificada e com a regressão logística nāo condicional.

\section{Comentários}

Observou-se consistência entre os resultados obtidos com a aplicaçāo das análises estratificada

Tabela 1- Casos e controles segundo sexo, idade e hábitos de beber $\theta$ fumar. Săo Paulo, 1981. Idade

Hábito

Hábito de beber de fumar

\begin{tabular}{|c|c|c|c|c|c|c|}
\hline \multirow[t]{4}{*}{ Feminino } & $\leq 57$ anos & Năo & $\begin{array}{l}\text { Năo } \\
\text { Sim }\end{array}$ & $\begin{array}{l}3 \\
-\end{array}$ & $\begin{array}{l}30 \\
15\end{array}$ & $\begin{array}{l}33 \\
15\end{array}$ \\
\hline & & Sim & $\begin{array}{l}\text { Nåo } \\
\text { Sim }\end{array}$ & 5 & $\begin{array}{l}14 \\
13\end{array}$ & $\begin{array}{l}14 \\
18\end{array}$ \\
\hline & $>57$ anos & Nåo & $\begin{array}{l}\text { Năo } \\
\text { Sim }\end{array}$ & $\begin{array}{l}2 \\
3\end{array}$ & $\begin{array}{c}41 \\
8\end{array}$ & $\begin{array}{l}43 \\
11\end{array}$ \\
\hline & & Sim & $\begin{array}{l}\text { Nào } \\
\text { Sim }\end{array}$ & 3 & $\begin{array}{l}6 \\
2\end{array}$ & $\begin{array}{l}6 \\
5\end{array}$ \\
\hline \multirow[t]{4}{*}{ Masculino } & $\leq 57$ anos & Năo & $\begin{array}{l}\text { Năo } \\
\text { Sim }\end{array}$ & 2 & $\begin{array}{c}9 \\
12\end{array}$ & $\begin{array}{c}9 \\
14\end{array}$ \\
\hline & & Sim & $\begin{array}{l}\text { Nāo } \\
\text { Sim }\end{array}$ & $\begin{array}{c}1 \\
40\end{array}$ & $\begin{array}{c}8 \\
58\end{array}$ & $\begin{array}{c}9 \\
98\end{array}$ \\
\hline & $>57$ anos & Năo & $\begin{array}{l}\text { Năo } \\
\text { Sim }\end{array}$ & : & $\begin{array}{c}6 \\
19\end{array}$ & $\begin{array}{c}6 \\
19\end{array}$ \\
\hline & & Sim & $\begin{array}{l}\text { Năo } \\
\text { Sim }\end{array}$ & - & $\begin{array}{c}4 \\
47\end{array}$ & $\begin{array}{c}4 \\
73\end{array}$ \\
\hline tal & & & & 85 & 292 & 377 \\
\hline
\end{tabular}


Tabela 3 - Análise estratificada para hábito de beber, controlando-se hábito de fumar.

\begin{tabular}{|c|c|c|c|c|c|c|}
\hline Estrato & & Caso & Controle & Total & Odds ratio & Intervalo com $95 \%$ de contiança \\
\hline Nảo fumante: & $\begin{array}{l}\text { Bebe } \\
\text { Năo bebe }\end{array}$ & $\begin{array}{l}1 \\
5\end{array}$ & $\begin{array}{l}32 \\
86\end{array}$ & $\begin{array}{l}33 \\
91\end{array}$ & 0,54 & $0,06-4,78$ \\
\hline Fumante: & $\begin{array}{l}\text { Bebe } \\
\text { Nāo bebe }\end{array}$ & $\begin{array}{c}74 \\
5\end{array}$ & $\begin{array}{l}120 \\
54\end{array}$ & $\begin{array}{c}194 \\
59\end{array}$ & 6,66 & $2,55-17,41$ \\
\hline Total & & 85 & 292 & 377 & $4,50^{*}$ & $2,11 \cdot 9,61$ \\
\hline
\end{tabular}

"Estimativa do odds ratio ponderado de Mantel-Haenszel $\left(O R_{n+1}\right)$

Teste de homogeneidade entre os estratos: $\chi_{1 q \mid}^{2}=4,27(p=0,04)$

Tabela 4 - Análise estratificada para hábito de beber, controlando-se sexo.

\begin{tabular}{llccccc} 
Estrato & & Caso & Controle & Total & Odds ratio & Intervalo com 95\% de confiança \\
\hline Feminino: & Bebe & 8 & 35 & 43 & 2,69 & $0,94 \cdot 7,71$ \\
& Nåo bebe & 8 & 94 & 102 & & \\
Masculino: & Bebe & 67 & 117 & 184 & 13,17 & $3,10-55,99$ \\
& Não bebe & 2 & 46 & 48 & & \\
\hline Total & & 85 & 292 & 377 & $6,28^{*}$ & $2,91-13,55$
\end{tabular}

* Estimativa do odds ratio ponderado de Mantel-Haenszel $\left(O R_{\mathrm{kH}}\right)$

Teste de homogeneidade entre os estratos: $\chi_{0,1}^{2}=3,03(p=0,08)$

Tabela 5 - Análise com regressăo logística nāo condicional para a variável hábito de beber, controlando-se (separadamente) hábito de fumar e sexo.

\begin{tabular}{clcc} 
Modelo & Variável de controle & $\begin{array}{c}\text { Hábito de beber } \\
\text { Odds ratio }\end{array}$ & Intervalo com $95 \%$ de confiança \\
\hline 1 & Hábito de fumar & 4,04 & $1,94-8,46$ \\
2 & Hábito de fumar & 0,54 & $0,06-4,78$ \\
& Hábito de fumar e hábito de beber & 12,39 & $2,55-17,40$ \\
3 & Sexo & 5,42 & $2,56-11,50$ \\
4 & Sexo & 2,69 & $0,94-7,71$ \\
& Sexo e hábito de beber & 4,90 & $0,82-29,38$
\end{tabular}

Tabela 6 - Análise estratificada para hábito de beber, controlando-se sexo e hábito de fumar.

\begin{tabular}{|c|c|c|c|c|c|c|c|}
\hline Sexo & Hábito de fumar & & Caso & Controle & Total & Odds ratio & $\begin{array}{c}\text { Intervalo com } \\
95 \% \text { de confiança }\end{array}$ \\
\hline Feminino & $\begin{array}{l}\text { Não fumante: } \\
\text { Fumante: } \\
\text { Não fumante: } \\
\text { Fumante: }\end{array}$ & $\begin{array}{r}\text { Bebe } \\
\text { Năo bebe } \\
\text { Bebe } \\
\text { Não bebe } \\
\text { Bebe } \\
\text { Năo bebe } \\
\text { Bebe } \\
\text { Não bebe }\end{array}$ & $\begin{array}{c}- \\
5 \\
8 \\
3 \\
1 \\
- \\
66 \\
2\end{array}$ & $\begin{array}{c}20 \\
71 \\
15 \\
23 \\
12 \\
15 \\
105 \\
31\end{array}$ & $\begin{array}{c}20 \\
76 \\
23 \\
26 \\
13 \\
15 \\
171 \\
33\end{array}$ & $\begin{array}{c}0 \\
4,09 \\
\text { indefinido } \\
9,74\end{array}$ & $0,93-17,92$ \\
\hline Total & & & 85 & 292 & 377 & $4.79^{\star}$ & $2,09-10,99$ \\
\hline
\end{tabular}

- Estimativa do odds ratio ponderado de Mantel-Haenszel $\left(O R_{N+1}\right)$

Teste de homogeneidade entre os estratos: năo fol possível (caselas vazias).

e logística. Quando o númẹro de variáveis a ser controlado simultaneamente é pequeno (uma ou duas), a análise estratificada é rápida, dispensa o uso de equipamentos eletrônicos e permite maior visibilizaçāo dos dados; resultados aparentemente paradoxais têm explicação quase que imediata. À medida em que o número de variáveis aumenta, a análise logística torna-se praticamente obrigatória, mesmo à custa de um possível distanciamento entre o pesquisador e os dados originais. Mas os dois tipos 
Tabele 7 - Análise com regressāo logística não condlcional para as variáveis hábito de beber, hábito de fumar e sexo (simultaneamente).

\begin{tabular}{lcc}
\hline Variáveis & Odds ratio & $\begin{array}{c}\text { Intervalo com } \\
95 \% \text { de confiança }\end{array}$ \\
\hline Hábito de beber & & \\
(variável principal) & 4,05 & $1,88-8,73$ \\
Hábito de fumar & 5,01 & $1,92-13,12$ \\
Sexo & 1,00 & $0,49-2,05$ \\
\hline
\end{tabular}

de abordagem não se excluem mutuamente ${ }^{11} . O$ modelo logístico e mais flexível, com maior poder de exploraçāo de variáveis. A existência de programas de microcomputador "amigáveis" e de uso livre, de abundância de cursos, de epidemiologistas com bom conhecimento de estatística, tornam cada vez mais conhecida e popular a análise logística. A análise estratificada será muitas vezes boa auxiliar para visibilização e compreensão das relações entre variáveis.

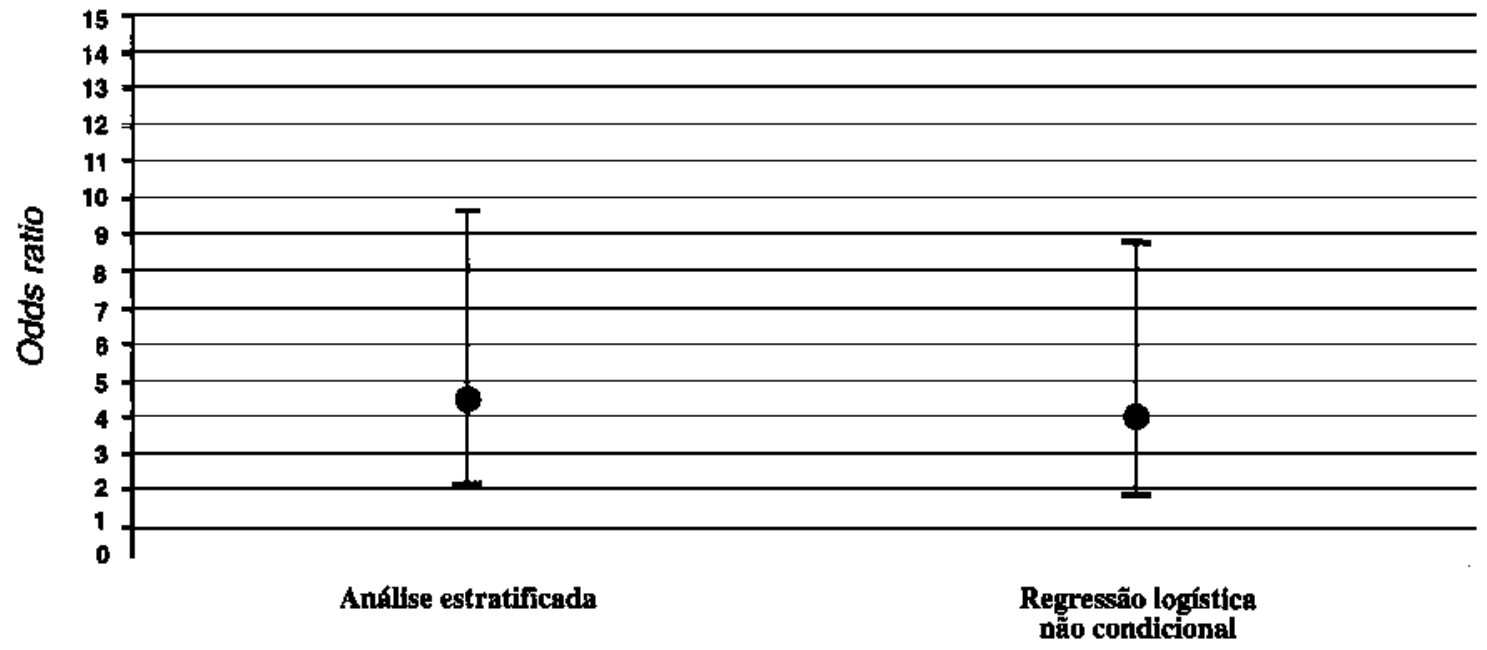

Tipo de Análise

Figura 1- Odds ratio e intervalo com $95 \%$ de confiança para hábito de beber, controlando-se hábito de fumar, segundo tipo de análise.

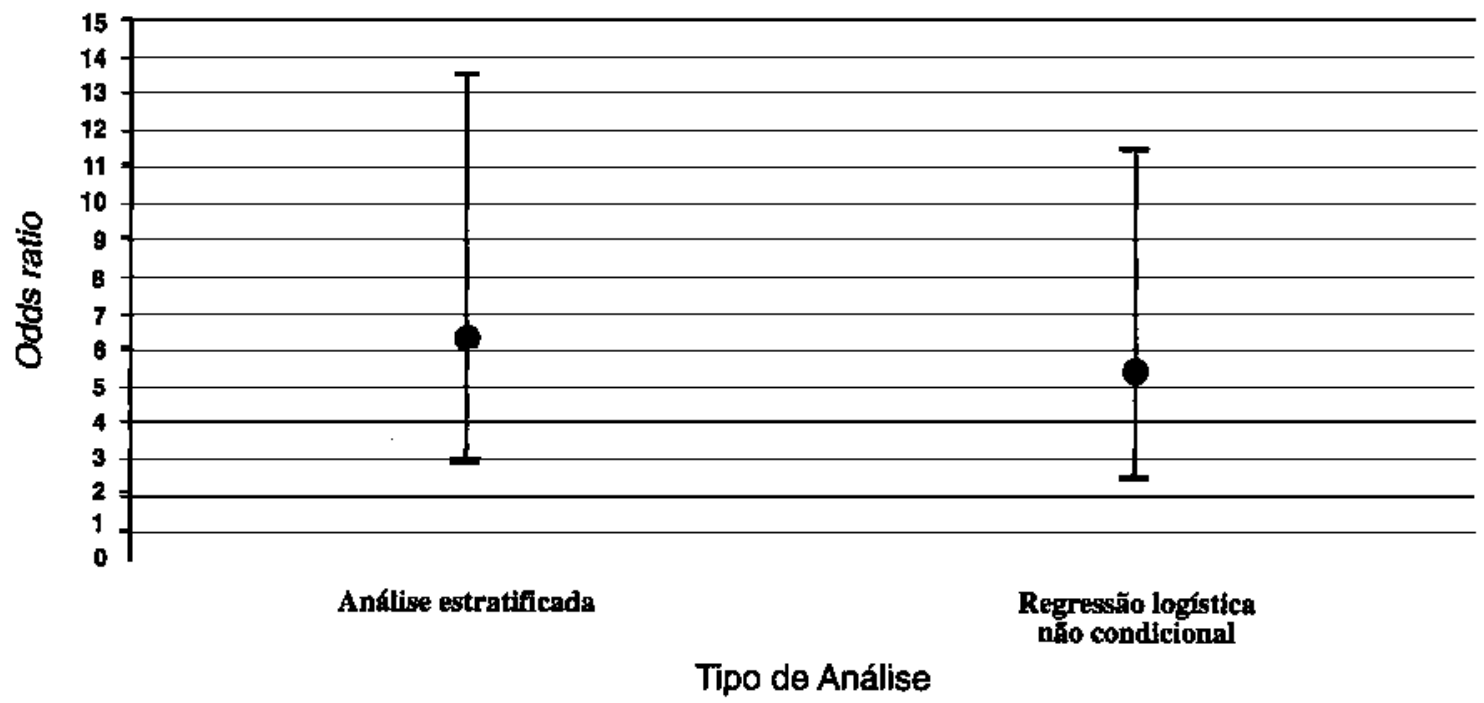

Figura 2 - Odds ratio e intervalo com $95 \%$ de confiança para hábito de beber, controlando-se sexo, segundo tipo de análise. 


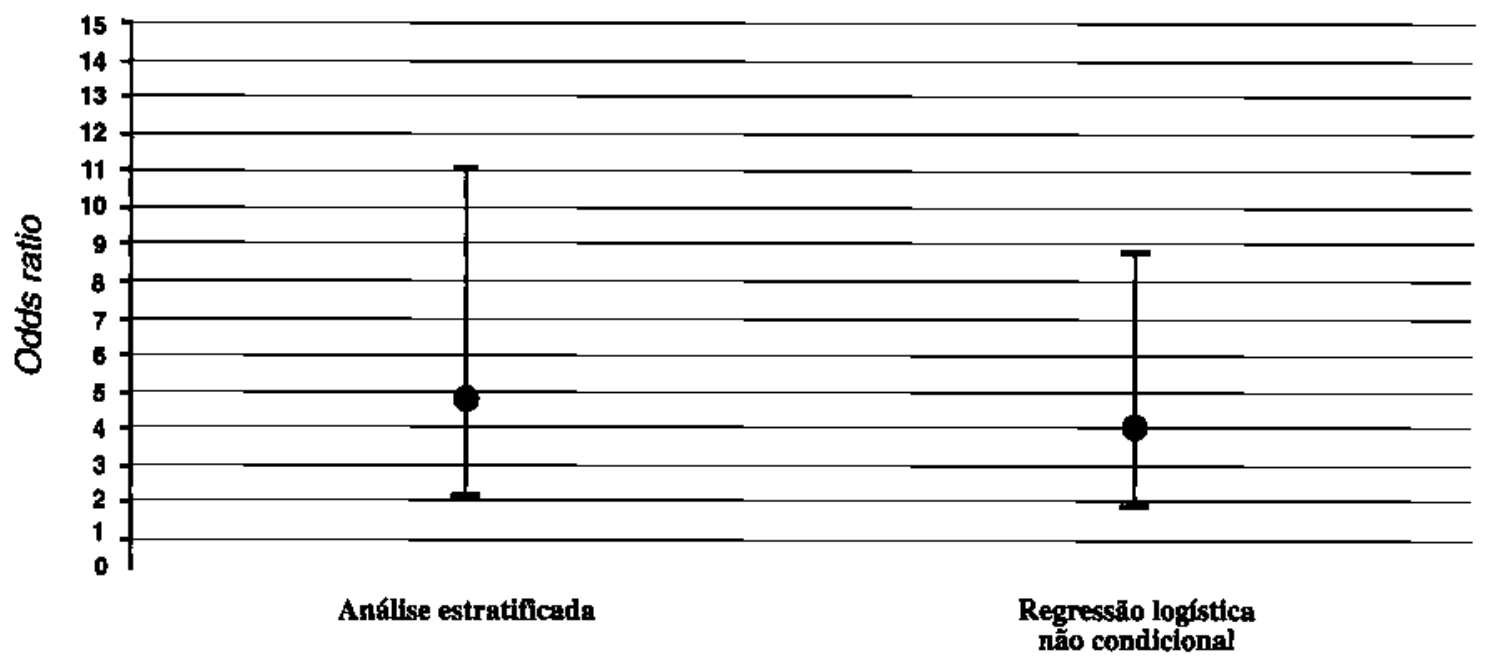

Tipo de Análise

Figura 3 - Odds ratio e intervalo com $95 \%$ de confiança para hábito de beber, controlando-se sexo e hábito de fumar, segundo tipo de análise.

\section{Agradeclmentos}

Aos relatores que apreciaram o manuscrito, pelas valiosas observaçōes e sugestzes.

\section{Referôncias Bibllográflcas}

1. ARMITAGE, P. \& BERRY, G. Statistical methods in medical research. 2nd. ed. Oxford, Blackwell Sci. Publ., 1987.

2. BRESLOW, N. E. \& DAY, W. Statistical methods in cancer research: the analysis of case-control studies. Lyon, 1980, v. 1 (IARC Scient. Publ. n. 32).

3. DEAN, J.; DEAN, A.; BURTON, A.; DICKER, R. Epi Infocomputer programs for epidemiology. Atlanta, Division of Surveilance and Epidemiologic Studies, Epidemiology Program Office, Center for Disease Control, 1990.

4. GREENLAND, S. Modelling and variables selection in epidemiologic analysis. Am. J. Epidemiol, 124:869-76, 1986.

5. HOSMER, D. M. \& LEMESHOW, S. Applied logistic regression. New York, John Wiley \& Sons, 1989.

6. GLMENO, S. G. A. et al. Fatores de risco para o cancer de esófago: estudo caso-controle em área metropolitana da regiāo Sudeste do Brasil. Rev. Saúde Pública, 29: 159$65,1995$.

7. MONCAU, J. E. C. Análise estratificada em estudos casocontrole. Såo Paulo, 1991. [Dissertação de MestradoFaculdade de Saúde Pública da USP].

8. MULTLR -A microcomputer program for multiple regression by condicional and uncondicional maximum likelihood methods. Am.J.Epidemiol, 129:439-44, 1989.

9. ROTHMAN, K. J. Modern epidemiology. Boston, Little and Co., 1986.

10. SCHLESSELMAN, J.J. Case-control studies - design, conduct, analysis. New York, Oxford University Press, 1982.

11. VANDENBROUCKE, J. P. Should we abandon statistical modeling altoghether? Am. J. Epidemiol., 126: 10-3, 1987.

\begin{abstract}
Data of a case-control study of esophageal cancer were used as an example of the use of multivariate analysis with stratification and logistic regression. Elghty-five cases and 292 controls were classified according to sex, age and smoking and drinking habits. The point estimates of the odds ratios were similar, and the techniques were considered complementary.
\end{abstract}

Multivariate analysis. Logistic regression. Case-control studies. 


\section{Apêndice}

\section{I: Análise Bruta}

Apresentação geral de uma Tabela formada por variáveis dicotômicas em estudo caso-controle

\begin{tabular}{lccc}
\hline \multirow{2}{*}{ Fator de estudo } & \multicolumn{2}{c}{ Condiçăa } & \\
\cline { 2 - 4 } & Caso & Controle & Total \\
\hline Exposto & $\mathrm{a}$ & $\mathrm{b}$ & $\mathrm{m}_{1}$ \\
Não exposto & $\mathrm{c}$ & $\mathrm{d}$ & $\mathrm{m}_{0}$ \\
\hline Total & $\mathrm{n}_{1}$ & $\mathrm{n}_{0}$ & $\mathrm{~T}$ \\
\hline
\end{tabular}

Odds ratio $=\hat{\mathrm{OR}}=\frac{\mathrm{ad}}{\mathrm{bc}}$

Intervalo de confiança para o odds ratio: $\exp [\ln (\hat{\mathrm{OR}}) \mp \mathrm{za} / 2 \sqrt{\mathrm{V}[\ln (\hat{\mathrm{OR}})]}]$

Variância do $\ln (\hat{O R})=V[\ln (\hat{O R})]=\frac{1}{a}+\frac{1}{b}+\frac{1}{c}+\frac{1}{d}$ (segundo Woolf)

Esperança de $a=E(a)=\frac{n_{1} m_{1}}{T}$

Variância de $a=V(a)=\frac{m_{1} n_{0} m_{0} n 1}{T^{2}(T-1)}$

Teste de hipóteses: $\chi_{\text {lgl }}^{2}=\frac{[\mathrm{a}-\mathrm{E}(\mathrm{a})]^{2}}{\mathrm{~V}(\mathrm{a})}$

\section{II: Análise Estratificada}

Odds ratio de Mantel-Haenszel $=\hat{O R} \quad M H=\frac{\sum_{i=1}^{1} \frac{a_{i} d_{i}}{T_{i}}}{\sum_{i=1}^{1} \frac{b_{i} c_{i}}{T_{i}}}$

Intervalo de confiança para o odds ratio $=\exp [\ln (\hat{\mathrm{OR}}) \mp \mathrm{za} / 2 \sqrt{\mathrm{V}[\ln (\hat{\mathrm{OR}})]}]$

$V\left[\ln \left(\hat{O R}_{M H}\right)\right]=\frac{\sum P_{i} R_{i}}{2\left(\sum R_{i}\right)^{2}}+\frac{\sum P_{i} S_{i}+\sum Q_{i} R_{i}}{2\left(\sum R_{i}\right)\left(\sum S_{i}\right)}+\frac{\sum Q_{i} S_{i}}{2\left(\sum S_{i}\right)^{2}}$,

onde $R_{i}=\frac{a_{i} d_{i}}{T_{i}} ; S_{i}=\frac{b_{i} c_{i}}{T_{i}} ; P_{i}=\frac{a_{i}+d_{i}}{T_{i}} ; Q_{i}=\frac{b_{i}+c_{i}}{T_{i}}$

Teste de homogeneidade entre I estratos:

$$
\chi_{\mathrm{I}-1 \mathrm{gl}}^{2}=\sum_{\mathrm{i}=1 \mathrm{~V}\left(\mathrm{~A}_{\mathrm{i}} \mid \hat{\mathrm{OR}}_{M H}\right)}^{\mathrm{I}}, \text { onde }
$$


$\mathrm{V}\left(\mathrm{A}_{1} \mid \hat{\mathrm{OR}}_{\mathrm{MH}}\right)=\frac{1}{\frac{1}{\mathrm{~A}_{1}}+\frac{1}{\mathrm{~B}_{1}}+\frac{1}{\mathrm{C}_{1}}+\frac{1}{\mathrm{D}_{1}}}$

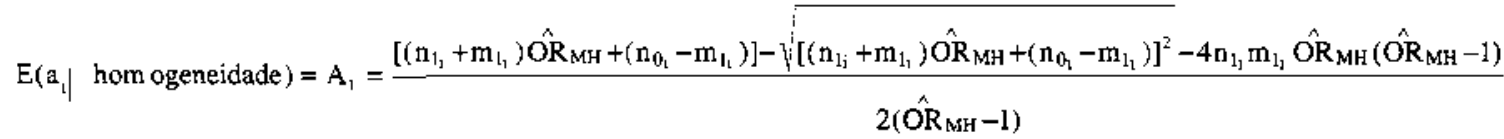

$\mathrm{B}_{\mathrm{i}}=\mathrm{m}_{\mathrm{li}}-\mathrm{A}_{\mathrm{i}} \quad \mathrm{C}_{\mathrm{i}}=\mathrm{n}_{1 \mathrm{i}}-\mathrm{A}_{\mathrm{i}} \quad \mathrm{D}_{\mathrm{i}}=\mathrm{n}_{0 \mathrm{i}}-\mathrm{B}_{\mathrm{i}}$

\section{III: Análise Logística}

Modelo sem Interação

Odds ratio $=\hat{\mathrm{OR}}=\exp (\hat{\beta})$

\section{Modelo com interação}

Odds ratio com interação:

estrato de nāo expostos à variável de controle: $\exp \left(\hat{\beta}_{\text {principal }}\right)$

estrato de expostos a variável de controle: $\hat{\mathrm{OR}}_{\text {principal }} \times \hat{\mathrm{OR}}_{\text {interą̧ão }}=\exp \left(\hat{\beta}_{\text {principal }}+\hat{\beta}_{\text {interação }}\right)$

Variância $=\mathrm{V}\left(\hat{\beta}_{\text {principal }}+\hat{\beta}_{\text {interaçåo }}\right)=\mathrm{V}\left(\hat{\beta}_{\text {principal }}\right)+\mathrm{V}\left(\hat{\beta}_{\text {interaçăo }}\right)+2 \operatorname{covariância~}\left(\hat{\beta}_{\text {principal }}, \hat{\beta}_{\text {interą̧ăo }}\right)$

Intervalo de confiança: $\exp \left[\left(\hat{\beta}_{\text {principal }}+\hat{\beta}_{\text {intera̧̧ăo }}\right) \pm z / 2 \sqrt{\text { Variância }}\right]$ 